\title{
Research on Control Method of Inverters for Large-scale Grid Connected Photovoltaic Power System
}

\author{
Zhuo Zhang, Hongwei Li \\ Power Supply Company of Zhengzhou, Henan, China \\ Email: zhang-zhuo@msn.com, lihongwei-6@163.com
}

Received 2013

\begin{abstract}
A grid-connected inverter controlling method to analyze dynamic process of large-scale and grid-connected photovoltaic power station is proposed. The reference values of control variables are composed of maximum power which is the output of the photovoltaic array of the photovoltaic power plant, and power factor specified by dispatching, the control strategy of dynamic feedback linearization is adopted. Nonlinear decoupling controller is designed for realizing decoupling control of active and reactive power. The cascade PI regulation is proposed to avoid inaccurate parameter estimation which generates the system static error. Simulation is carried out based on the simplified power system with large-scale photovoltaic plant modelling, and the power factor, solar radiation strength, and bus fault are considered for the further research. It's demonstrated that the parameter adjustment of PI controller is simple and convenient, dynamic response of system is transient, and the stability of the inverter control is verified.
\end{abstract}

Keywords: Large-scale Photovoltaic Grid-connected; Dynamic Feedback Linearization; Nonlinear Decoupling; Cascade Connection PI Control

\section{Introduction}

The safety and economy of power system are affected directly by transformer running states, which play an important role in network. According to the survey, the total transformer loss of about $8 \%$ of electricity generation, and distribution transformer loss is accounted to about $60 \sim 80 \%$ of the entire distribution grid [1, 2]. Nowadays, a large number of frequency electrical appliances and devices sorted as non-linear loads in industrial and lives have become increasingly universal, which have led to harmonic pollution to system and brought about adverse effects including increased wear and tear, abnormal temperature rise, insulation reduced life expectancy shortened to transformers and other electromagnetic equipments[3]. Therefore, non-linear load loss calculation and analysis for transformer has been concerned by the very important.

Traditional transformer loss calculation includes theoretical analysis and experimental measurements. In reference [4], curve fitting method applied, and the harmonics equivalent parameters are calculated with large number of experimental information as to harmonic loss by superposition principle. Core saturation is not put into consideration, and THD for different parameters can be corrected. IEEE standards with experimental measurements and operating experience data to calculate the harmonic losses [5], but DC resistance loss is obtained roughly, and the eddy current and stray losses are not distributed considerably. Besides, the conservation is mentioned [6]. The document [7] studied the curve fitting, and brought out better method when dealing with high frequency harmonic problem.

As studied above, equivalent parameter model has been built, and harmonic loss is analyzed in this paper by considering the winding conductor frequency-dependent characteristics with model parameters and the non-linear superposition.

\section{Winding Harmonic Model}

Transformer total loss includes the copper loss, iron loss and other stray loss, and copper loss of windings is divided into dc loss and winding eddy loss. The total loss is consisted of dc transformer winding loss, winding eddy current loss and other stray loss since iron loss has been ignored in load operation [8].

The harmonic equivalent circuit of transformer has been shown in Figure 1. In which, $R_{h(1)}, R_{h(2)}, X_{h(1)}, X_{h(2)}$ is winding equivalent resistance values and reactance values at order $h$ respectively; $R_{h(\mathrm{~m})}$ and $X_{h(\mathrm{~m})}$ is magnetic resistance and reactance.

Without regard to core saturation, groups of equivalent parameters information are acquired through no-load, 


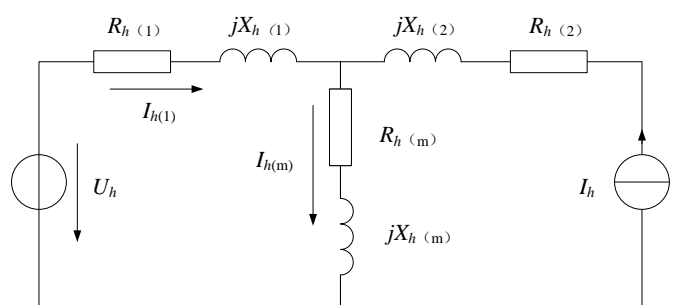

Figure 1. The harmonic equivalent circuit of transformer.

short circuit test to test different harmonics effect to the transformer. The superposition principle is employed to the harmonic losses while the algebraic sum of the value of consumed energy. Characterized by curve fitting the winding resistances under different frequency have been expressed as harmonic equivalent nonlinear parameters.

$$
\begin{gathered}
R_{h(1,2)}=\alpha e^{\beta h} R_{1(1,2)} \\
X_{h(1,2)}=\left(a_{0}+a_{1} h-a_{2} h^{2}\right) X_{1(1,2)}
\end{gathered}
$$

The parameters in the formula are interpreted as follows:

$R_{1(1,2)}, R_{h(1,2)}$ - fundamental and $\mathrm{h}$ harmonic equivalent value of former and vice side winding resistance;

$X_{1(1,2)}, X_{h(1,2)}$ - fundamental and h harmonic equivalent value of winding reactance;

$\alpha$ - coefficient for exponential term;

$\beta$ - polynomial fitting exponent.

Harmonic loss is expressed as:

$$
P_{h}=3 I_{h(1)}^{2} R_{h(1)}+3 I_{h(2)}^{2} R_{h(2)}
$$

The total transformer losses are shown:

$$
P_{T}=\sum_{h=1}^{h=h_{\max }}\left(3 I_{h(1)}^{2} R_{h(1)}+3 I_{h(2)}^{2} R_{h(2)}\right)
$$

IEEE / ANSI C57.110 standard made definition of the total transformer losses under rated condition [9]:

$$
P_{L L-R}=I_{R}^{2} R_{d c}+P_{E C-R}+P_{O S L-R}
$$

where, $P_{L L-R}$ is the total loss, $R_{D C}$ is the DC resistance, $P_{E C-R}$ is rated winding eddy current losses, and $P_{\text {OSL-R }}$ is the other stray loss. With non-linear loads, the following definition is achieved [10]:

$$
\begin{gathered}
F_{H L}=\frac{\sum_{h=1}^{h_{\max }} I_{h}^{2} h^{2}}{\sum_{h=1}^{h_{\max }} I_{h}^{2}}=\frac{\sum_{h=1}^{h_{\max }}\left[\frac{I_{h}}{I_{1}}\right]^{2} h^{2}}{\sum_{h=1}^{h_{\max }}\left[\frac{I_{h}}{I_{1}}\right]^{2}} \\
F_{H L-S T R}=\frac{\sum_{h=1}^{h_{\max }} I_{h}^{2} h^{0.8}}{\sum_{h=1}^{h_{\max }} I_{h}^{2}}=\frac{\sum_{h=1}^{h_{\max }}\left[\frac{I_{h}}{I_{1}}\right]^{2} h^{0.8}}{\sum_{h=1}^{h_{\max }}\left[\frac{I_{h}}{I_{1}}\right]^{2}}
\end{gathered}
$$

$F_{H L}$ is harmonic loss factor for winding eddy currents. $F_{H L-S T R}$ is the other stray loss factor. It is not considered about the model parameters of nonlinear problems, harmonic losses are calculated by overlapping the corresponding value.

Total loss is defined as below:

$$
P_{L L}=I_{R}^{2} R_{d c}+F_{H L} P_{E C-R}+F_{H L-S T R} P_{O S L-R}
$$

\section{Equivalent Model Frequency Characteristic}

Transformer eddy current loss is generated by alternating magnetic flux, followed with thermal effect and magnetic effect, which is shown in Figure 2.

The eddy currents or magnetic diffusion equation can be derived in quasi-static magnetic field (MQS).

$$
\left\{\begin{array}{l}
\nabla^{2} \boldsymbol{H}=\mu \gamma \frac{\partial \boldsymbol{H}}{\partial t} \\
\nabla^{2} \boldsymbol{E}=\mu \gamma \frac{\partial \boldsymbol{E}}{\partial t} \\
\nabla^{2} \boldsymbol{J}=\mu \gamma \frac{\partial \boldsymbol{J}}{\partial t}
\end{array}\right.
$$

The distributed electric and magnetic field capacity are fixed as the magnetic center of the distribution known:

$$
\left\{\begin{array}{l}
\dot{H}_{z}(x)=\frac{\dot{H}_{0}}{\operatorname{ch}(K a)} \operatorname{ch}(K x) \\
\dot{E}_{y}(x)=-\frac{K \dot{H}_{0}}{\gamma \operatorname{ch}(K a)} \operatorname{sh}(K x)
\end{array}\right.
$$

In order to facilitate analysis of conductor in the electromagnetic field distribution, the following assumptions are proposed:

1) As $l>>a, b>>a$, it is assumed that conductor dimension along $y$ or $z$ direction is much larger than $x$ direction. Otherwise, the geometric size of the thickness is much smaller. $\boldsymbol{E}, \boldsymbol{H}$ and other field quantity can be approximated as function of $x, y$ and $z$ independently.

2) Conductor is supposed passed by sinusoidal magnetic field and $\mathrm{B}$ along the $z$ direction. Then the eddy current in $x-y$ section was a closed path, but not spread along direction $z$. As $b>>a$ mentioned above, the edge effect of y plane can be ignored. That is, $\boldsymbol{E}(\boldsymbol{J})$ only has the component of $y, E_{y}, J_{y}$, while $\mathrm{H}$ only contains $H_{z}$.

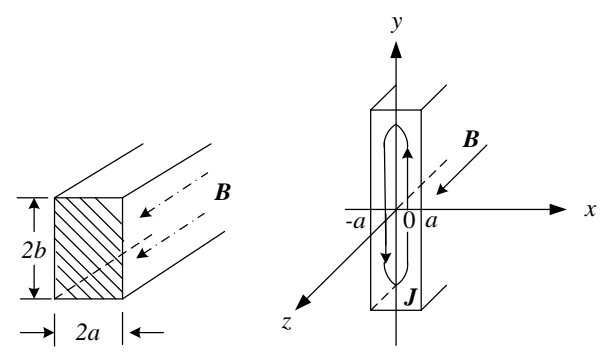

Figure 2. Conductor eddy current distribution. 
Current density analysis of electromagnetic wave propagation in the conductor:

$$
\dot{J}_{z}=C_{1} e^{-K x}+C_{2} e^{K x}=-\frac{\dot{B}_{0} K}{\mu} \operatorname{sh}(K x)
$$

Attenuation of field penetration depth $d$ is estimated with the expression:

$$
\left\{\begin{array}{l}
K=1 / d \\
\dot{J}_{z}=\dot{J}_{0} \operatorname{sh}(K x)
\end{array}\right.
$$

The harmonics generated in the power density of ferromagnetic materials changes as shown below:

Magnetic flux pass through the core along parallel position of laminations, takes $\mu_{1}=1000 \mu_{0}, \gamma_{1}=10^{7} \mathrm{~S} / \mathrm{m}, a=$ $5 \times 10^{-4} \mathrm{~m}$. In the frequency case, the electromagnetic field penetration depth is of about $7 \times 10^{-4} \mathrm{~m}$, while silicon steel sheet is always designed in thickness of $3 \times 10^{-4} \mathrm{~m}$. Winding parameter takes $\mu_{2} \approx 10^{-3} \mu_{1}, \gamma_{2} \approx 10 \gamma_{1}, a=5 \times$ $10^{-3} \mathrm{~m}$, and the magnetic field perpendicular to the circle direction. Harmonic currents under high frequency through the conductor reduce the amplitudes, and $d=$ $(2 / \omega \mu \gamma)^{0.5}$, The equivalent $R$ has changed accordingly to $d$ or $\omega^{0.5}$ proportionally. It is known that magnetic field and eddy current in the conductor are not uniformly distributed. Correction factor is brought forward as eddy effect taken into account:

$$
K_{H L}=\sum_{h=1}^{h=h_{\max }}\left[\frac{\omega_{h}}{\omega_{R}}\right]^{\alpha} T H D_{h}^{2}
$$

Take the variables in the formula, $K_{H L}$ factor for consideration, $\omega_{h}$ for the $\mathrm{h}$ harmonic angular frequency, $\omega_{R}$ to fundamental wave angular frequency, $\mathrm{THD}_{h}$ for the total $h$ distortion harmonics, $\alpha$ for the frequency- dependent exponent. Non-linear load transformer losses are:

$$
\left\{\begin{array}{l}
P_{E C}^{\prime}=\left(1+K_{H L}\right) \times P_{E C-R} \\
P_{L L}^{\prime}=P_{d c}+P_{E C}^{\prime}+P_{O S L}
\end{array}\right.
$$

IEEE / ANSI C57.110 standard refers only to non- linear load current increase in RMS, thus DC resistance

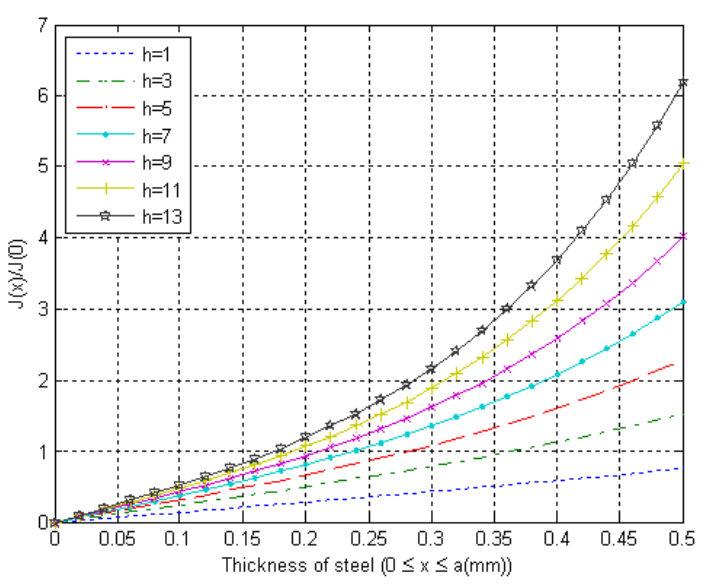

Figure 3. Current density of the harmonic components.

loss will be increased correspondingly. It is concluded that the loss calculation is rough under harmonic for non-linear condition. Therefore, the amended form as follows:

$$
P_{d c}=\sum_{h=1}^{h=h_{\max }}\left(I_{1 h}^{2} R_{d c 1}+I_{2 h}^{2} R_{d c 2}\right)
$$

\section{Methods Comparation and Simulation}

To facilitate the comparative analysis, take the literature [3] model as an example. Specific parameters and data are listed in the Table 1, $\mathbf{2}$.

For simulation, the actual heat pump system has been taken as example. Frequency control device that produces $6 k \pm 1(k=1,2, \ldots)$ characteristic harmonics is a typical harmonic source in power system and THD is about $20 \%$ [11]. The circuit principle is shown in Figure 4. Transformer model has referred the data provided in Table $\mathbf{1 .}$ Common frequency converter is consisted of the threephase bridge uncontrolled rectifier on the rectifier side and the PWM control on the inverter side. And squirrel cage induction motor is exploited as load.

Data of Table 2 is valid in formula (1) (3) for transformer losses calculation, the result and simulation value are gained and compared.

Table 1. Transformer parameters.

\begin{tabular}{cccc}
\hline Rated power & $50 \mathrm{kVA}$ & Load losses & $1250 \mathrm{~W}$ \\
\hline high voltage $(\mathrm{HV})$ & $20 \mathrm{kV}$ & HV current & $1.44 \mathrm{~A}$ \\
low voltage $(\mathrm{LV})$ & $400 \mathrm{~V}$ & LV current & $72 \mathrm{~A}$ \\
HV winding resistance & $121.5 \Omega$ & LV winding resistance & $0.03 \Omega$ \\
\hline
\end{tabular}

Table 2. The value of the load harmonic current.

\begin{tabular}{cccccccc}
\hline$h$ & 1 & 5 & 7 & 11 & 13 & 17 & 19 \\
\hline$I_{h}(\mathrm{~A})$ & 70.2 & 12.312 & 7.7760 & 3.1680 & 2.0160 & 1.0800 & 0.7056 \\
\hline
\end{tabular}




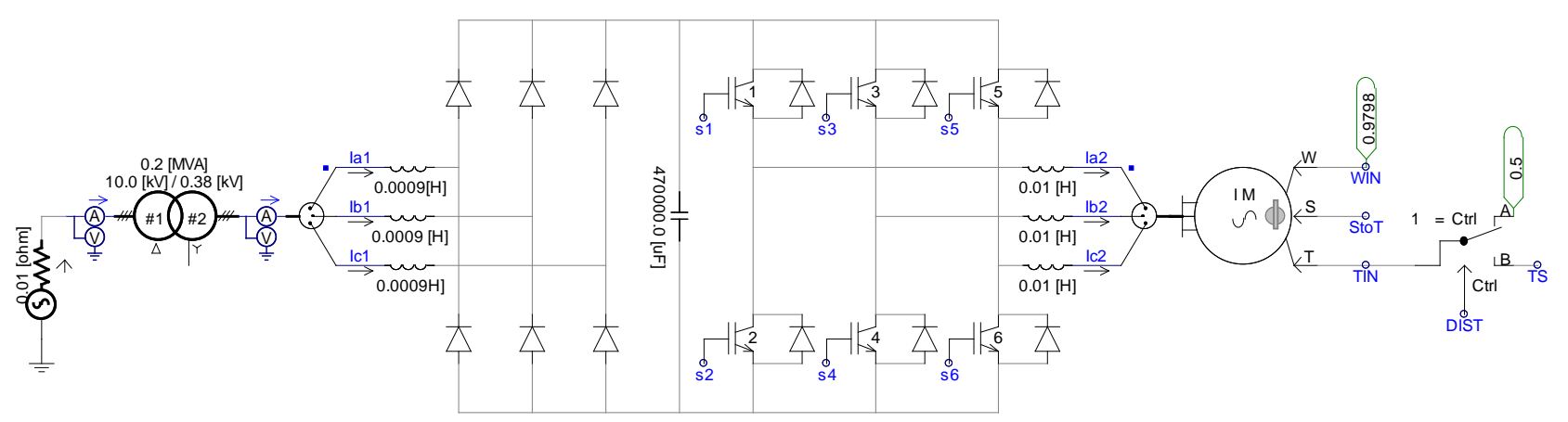

Figure 4. Adjustable speed drive system simulation circuit.

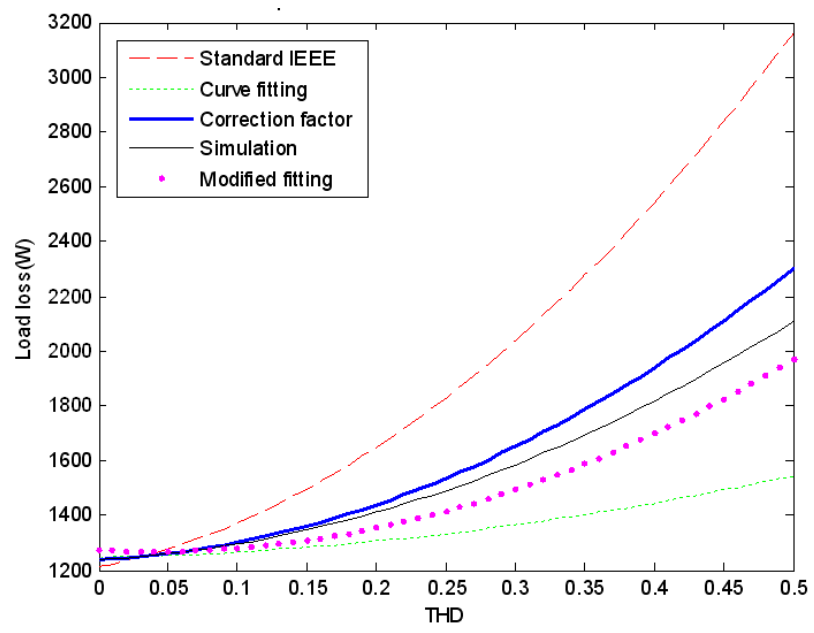

Figure 5. Comparison the results of various calculation methods.

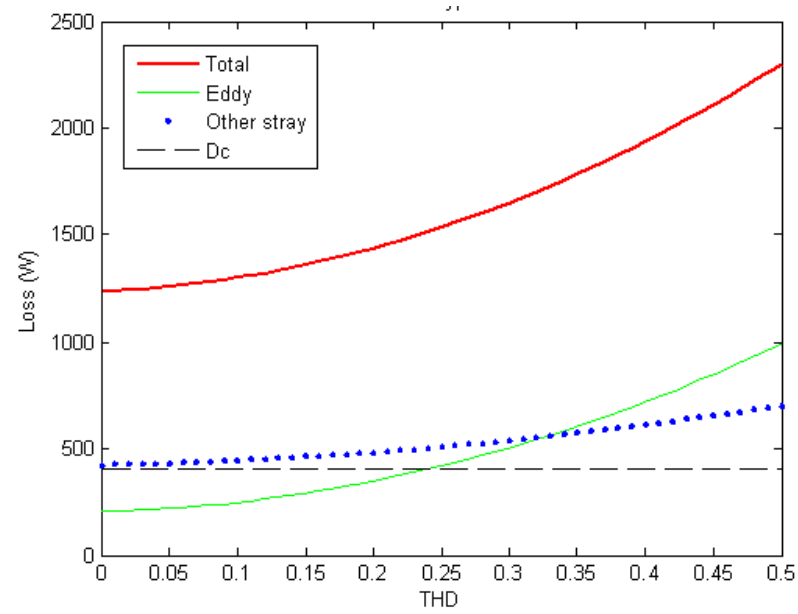

Figure 6. Types of loss and the relationship with THD.

Table 3. Losses calculation of methods and simulation.

\begin{tabular}{lccccc}
\hline $\begin{array}{c}\text { Curve } \\
\text { fitting }\end{array}$ & $\begin{array}{c}\text { Modified } \\
\text { fitting }\end{array}$ & $\begin{array}{c}\text { Standard } \\
\text { IEEE }\end{array}$ & $\begin{array}{c}\text { Revising } \\
\text { factor }\end{array}$ & Simulation \\
\hline$P_{L L}$ & $1313 \mathrm{~W}$ & $1398 \mathrm{~W}$ & $1670 \mathrm{~W}$ & $1454 \mathrm{~W}$ & $1421 \mathrm{~W}$ \\
\hline
\end{tabular}

Exploiting this method calculated transformer losses of different THD, the results of calculation are shown in Figure 5.

In the case that transformer with a non-linear load, the losses increase is mainly caused by the winding eddy current losses of harmonic, which also determines the upward trend of the total loss. Figure 6 makes it visible, which is consistent with the IEEE standard.

Revising factor of loss calculation is obtained from the above methods and simulation comparative analysis, which take into account the frequency harmonics under different winding parameters and the equivalent nonlinear superposition of harmonic loss calculation. Complexity is the same to the IEEE standard and accuracy is better than the IEEE standard, curve fitting, and the results of calculation closer to realistic situations.

\section{Conclusions}

As equivalent parameter model has been established, and the harmonic winding model frequency-dependent characteristics analyzed, transformer winding eddy current equations is derived under high-changed harmonic. Compared with traditional methods, this method has the following advantages:

1) Harmonic current of the conductor in the transmission will produce a frequency-dependent effect, which has been analyzed from the magnetic field. That is, the eddy current in the conductor magnetic field is not uniformly distributed.

2) Taking into account the different frequency harmonic wave windings of the equivalent nonlinear parameter and the harmonic superposition calculation of loss, the exponent of frequency change has been amended. Thus, $K$ and $F$ coefficient are avoided causing conservative calculations, and result is more reasonable.

\section{REFERENCES}

[1] L. W. Pierce, "Transformer Design and Application Consideration for non Sinusoidal Load Currents,” IEEE Trans, on Industry Applications, Vol. 2, No. 3, 1996, pp. 33-645. 
[2] C. J. Liu and R. G. Yang, "Calculation and Analysis of Transformer's Harmonic Loss,” Power System Protection and Control, 2008.

[3] M. B. B. Sharifian, J. Faiz, S. A. Fakheri and A. Zraatparvar, "Derating of Distribution Transformers for Non-sinusoidal Load Currents using Finite Element Method,” Vol. 25, No. 7, 2003, pp. 754-757.

[4] E. B. Makram, R. L. Thompson and A. A. Girgis, “A New Laboratory Experiment for Transformer Modeling in the Presence of Harmonic Distortion using a Computer Controlled Harmonic Generator," IEEE Trans Power System,1988. doi:10.1109/59.193007

[5] IEEE Recommended Practice for Establishing Transformer Capability When Supplying Nonsinusoidal Load Currents, ANSI/IEEE Standard C57, 2008, pp. 110-2008.

[6] D. Yildirim and E. F. Fuchs, "Measured Transformer Derating and Comparison with Harmonic Loss Factor (FHL) Approach," IEEE Transactions on Power Deliwery, Vol. 15, 2000, pp. 186-191.

doi:10.1109/61.847249
[7] P. Li, G. D. Li, Y. H. Xu and S. J. Yao, "Methods Comparation and Simulation of Transformer Harmonic Losses," 2010,

[8] E. F. Fuchs, D. Yildirim and W. M. Grady, "Measurement of Eddy-current Loss Coefficient PEC-R, Derating of Single-phase Transformers and Comparison with K-factor approach,” IEEE Trans. on Power Delivery, Paper 99WM104, to be published.

[9] IEEE Recommended Practice for Establishing Transformer Capability when Supplying Non-sinusoidal Load Currents, ANSUEEE Sfandard C57, 1986, p. 110.

[10] S. Tao, X. N. Xiao, "Comparing Transformer Derating Computed Using the Harmonic Loss Factor FHL and K-Factor," Advanced Technology of Electrical Engineering and Energy, 2008.

[11] I. Faiz, at al., "Research Repotl on Effect of Non-sinusoidal Loads upon Distribution Transformers and Wmtion Factor Estimation for Optimal Qeration of Transformer- Part l,” (in Persian), Azarbaijon Regional Elecm'cig Company, TabriL Iran, Spring 2001. 